Voix et Images

volxetimages

\title{
L'impossible est une frontière toujours reculante
}

\section{Robert Major}

Volume 22, numéro 3 (66), printemps 1997

Gilbert Langevin

URI : https://id.erudit.org/iderudit/201329ar

DOI : https://doi.org/10.7202/201329ar

Aller au sommaire du numéro

Éditeur(s)

Université du Québec à Montréal

ISSN

0318-9201 (imprimé)

1705-933X (numérique)

Découvrir la revue

Citer cet article

Major, R. (1997). L'impossible est une frontière toujours reculante. Voix et Images, 22(3), 601-607. https://doi.org/10.7202/201329ar d'utilisation que vous pouvez consulter en ligne.

https://apropos.erudit.org/fr/usagers/politique-dutilisation/ 


\title{
Essai
}

\section{L'impossible est une frontière toujours reculante}

\author{
Robert Major, Université d'Ottawa
}

Il paraît que Napoléon avait rayé le mot "impossible " de son vocabulaire et qu'on devrait au grand homme, parmi les nombreuses phrases historiques et sentences cornéliennes qu'on lui attribue, la déclaration superbe et péremptoire que "impossible n'est pas français *.

Le mot fut donc banni par l'Empire et déclaré inexistant. Peut-être ne faut-il pas chercher plus loin les raisons de la remarquable fortune littéraire du mot et de la chose! En effet, naquirent, à l'époque même de Napoléon, toute une catégorie de récits qui se braquaient sur l'impossible, l'invraisemblable et l'inimaginable, procurant de multiples frissons à leurs lecteurs et faisant basculer les frontières mêmes du réel. Jusqu'alors, tout était possible à la guerre et en politique, mais la littérature se voulait raisonnable ou à tout le moins vraisemblable. Or, voici que même la littérature s'éloignait de ses voies traditionnelles, codifiées par Aristote. À l'impossible, chacun, même les écrivains, était maintenant tenu!

Ces récits, populaires à partir de la fin du XvIII $^{\mathrm{e}}$ siècle, ne furent pas toujours le fait d'obscurs scribouilleurs. Par exemple, Victor Hugo, dont Les travailleurs de la mer ont fourni le titre de cette chronique, s'évertuait souvent à faire reculer les frontières du réel et s'amusait à promener ses lecteurs sur les marches éloignées de la raison. Il était fasciné par le monstrueux et l'inexplicable. Pour lui, le surnaturalisme était un des trois horizons du Génie.

Notre fin de siècle ressemble à cette autre. Il est beaucoup question, depuis quelque temps, de la littérature fantastique. Le phénomène sort des chapelles d'initiés et devient l'objet d'études universitaires et de groupes de recherches ${ }^{1}$. En témoignent deux titres récents, portant sur des corpus québécois: La logique de l'impossible. Aspects du discours fantastique québécois de Michel Lord ${ }^{2}$, et La nouvelle fantastique québécoise de 1960 à 1985. Entre le basard et la fatalité de Lise Morin ${ }^{3}$. Cette institutionnalisation universitaire est une consécration qui réjouira les amateurs du genre. Pour les autres - dont le signataire de cette chronique, peu habitué à ce monde particulier et même plutôt réfractaire aux monstres sulfureux et à tout ce qui déborde des cadres familiers et rassurants de la raison raisonnante - , l'occasion est sans doute bonne pour une incursion timide dans le monde de l'étrange. 


\section{$\stackrel{*}{* *}$}

Pour Michel Lord, et c'est son incipit, -depuis maintenant plus de deux siècles, le récit fantastique fait partie intégrante de l'histoire littéraire occidentale " (9). Peut-être l'épithète * intégrante " sera-t-elle jugée excessive, car l'auteur reconnaît lui-même que ce genre de discours apparaît et disparaît à différents moments. Dans la mesure, toutefois, où le fantastique semble avoir une fonction précise, celle de amouler dans une forme artistique des interrogations relatives à la croyance en l'improbable qui circulent dans la pensée individuelle et sociale " (10), et qu'il exerce cette fonction depuis deux siècles, sans doute convient-il d'accepter l'affirmation.

Du moins, pour ce qui est du Québec, faut-il reconnaître que le surnaturel et le fantastique sont présents dès le premier roman ( $L^{\prime} i n$ fluence d'un livre, 1837); de plus, le premier mouvement littéraire leur fait une large place, avec les contes de Casgrain, Fréchette, Taché, Lemay. Difficile, donc, de mettre en question l'opportunité d'une étude rigoureuse du fantastique québécois. Toutefois, cette inspiration fantastique disparaît à la fin $\mathrm{du} \mathrm{XIX}^{\mathrm{e}}$ siècle pour renaître vers 1960. Dans son introduction historique, Michel Lord ne s'interroge pas sur les raisons de cette disparition ni sur celles du retour; il se contente d'affirmer que $\cdot$ des raisons sociopolitiques et culturelles très complexes " (12) expliquent la renaissance, sans dire lesquelles et sans justifier la relation établie avec la Révolution tranquille. En réalité, peu lui chaut de laisser ainsi ses lecteurs sur leur faim: son propos est ailleurs.
Certes, il se livre à une périodisation que d'aucuns jugeront tatillonne (trois périodes distinctes entre 1960 et 1985 , et une autre depuis, c'est sans doute beaucoup, pour une production somme toute restreinte...), mais ce qui lui importe n'est pas l'histoire ni les explications sociohistoriques. Michel Lord s'interroge sur la spécificité québécoise du récit fantastique, d'une part, et pour ce faire il veut analyser le fonctionnement formel de ce type de récit, dans une - double perspective narratologique et générique " (23). Son objectif est d'en arriver à une conceptualisation du fantastique en tant que pratique générique" (23). Le fantastique est-il un genre? Si oui, quelles en sont les marques?

Le corpus analysé est formé d'une œuvre de cinq nouvellistes différents ${ }^{4}$, hommes et femmes (Claude Mathieu, André Carpentier, Michel Bélil, Daniel Sernine, Claudette Charbonneau-Tissot); ces œuvres couvrent deux décennies (19601985), abordent des contenus différents et incarnent des formes canoniques ou innovatrices du fantastique. L'échantillonnage est réduit mais suffisant, selon Michel Lord, pour permettre une démarche déductive, donc scientifique, puisqu'elle sera axée sur la cohérence interne de la théorie élaborée. Restera ensuite à vérifier les hypothèses sur un corpus plus étendu.

L'analyse, toutefois, est précédée d'une section théorique où sont passées en revue les différentes positions des théoriciens les plus importants du genre et où Michel Lord précise ses hypothèses, son modèle et sa méthode. "L'approche philosophique. de Louis Vax, "l'approche structura- 
liste de Tzvetan Todorov et sla poétique "d'Irène Bessière lui inspirent un certain nombre de réserves, mais aussi des idées et des pistes qu'il fait siennes. Il en tire une hypothèse de travail qui serait *la pierre angulaire de la poétique du récit fantastique : - [L]'esthétique du questionnement sur l'étrange forme la matière/manière du récit fantastique.. (40) Il importe alors de cerner la mise en texte de ce questionnement. L'étude de la mise en discours du fantastique s'effectuera donc par l'analyse de trois composantes du récit, qui deviendront du fait même les trois parties de l'étude: les types d'action ou les fonctions transformationnelles du récit (le système narratif ou la logique narrative), qui permettent de déterminer où se situe l'inscription de l'étrange; les procédés descriptifs de l'espace-temps et des acteurs, qui participent à la formalisation du fantastique; les types d'échanges verbaux (dialogismes, argumentations, langages, communication) par lesquels sont questionnés et problématisés les croyances et les événements vécus. À trois niveaux, donc, l'improbable est à la fois et successivement affirmé et contesté par différentes marques textuelles du récit.

Dans la systématisation de Michel Lord et pour les besoins de son étude, le fantastique est un hypogenre (c'est-à-dire une esthétique, au même titre que le tragique, le réalisme, le satirique, l'humoristique...) rattaché à un genre (ou catégorie de récit: dans ce cas, la nouvelle) qui, lui, relève d'un hypergenre (ou grande catégorie: dans ce cas, l'hypergenre narratif). Cette hiérarchisation lui permet d'examiner le sousgenre fantastique -comme pratique spécifique d'écriture " (51) et d'affirmer, par exemple, au début de la première partie:

Le fantastique est un hypogenre qui se réalise d'abord et avant tout dans des genres comme le roman, le conte et la nouvelle, par le recours aux procédés propres à l'hypergenre narratif. Dans ce contexte, il est normal d'examiner l'hypothèse suivante: l'ancrage premier du discours fantastique se trouve dans la narrativité, c'est-à-dire dans le processus complexe de transformation d'au moins un acteur à travers le déroulement d'une série d'actions ou d'événements, provoqués ou subis, axés autour de la représentation d'un phénomène et racontés par un ou plusieurs narrateurs. Or, depuis les travaux de Propp, de Greimas et de Bremond, nous savons que la narrativité opère selon des règles, selon une logique qui procède d'un certain formalisme actionnel, le récit d'événements ayant besoin d'être soutenu par des procédés de narration, dans le fantastique autant - sinon plus - que dans toute autre forme de récit. (59, souligné dans le texte)

Cette citation est révélatrice de la méthode de Michel Lord et de sa démarche déductive. Chacune des trois grandes parties de l'étude sera ainsi précédée d'un chapitre théorique qui pose les assises, précise les notions utilisées et indique le découpage qui sera effectué. Propp, Greimas, Bremond, mais surtout Jean-Michel Adam (Le texte narratif, Le récit) sont mis à contribution pour l'analyse du système narratif; les travaux de Philippe Hamon et d'Adam et Petitjean servent de fondement à l'analyse du descriptif; Bakhtine, surtout, est la caution théorique pour l'étude du dialogisme. 
Cette façon de procéder confère à l'étude de Michel Lord une grande qualité méthodologique et une utilité certaine pour l'analyse de tout texte narratif, qu'il soit de "l'hypogenre* fantastique ou non. Par ailleurs, cette démarche donne aussi à l'analyse une dimension plutôt sèche et austère. Rançon de la rigueur, sans doute. La scientificité s'acquiert à ce prix, semble-t-il. Mais le classement, l'extrême segmentation, voire la compartimentation en macropropositions, concaténations, séquences, modalités, paradigmes, chronotypes, procédures d'ancrage et de désancrage, prédicats éthologiques, etc., écrasent les textes eux-mêmes, il me semble.

Les spécialistes du récit apprécieront cette étude savante pour sa grande rigueur. Les théoriciens en herbe, également, car ils y trouveront une présentation systématique et une explication des aspects les plus importants d'une analyse formelle d'un récit. Les propagandistes du sousgenre fantastique seront heureux de cette mise en valeur des précédés rigoureux mis en ouvre par la machine textuelle de l'écrivain fantastiqueur. Quant aux amateurs du genre et aux simples curieux, il n'est pas sûr qu'ils y trouvent leur compte. Mais ils étaient hors champ, de toute façon. Car ce n'est pas la logique de l'impossible qui les intéresse, mais le subtil frisson ressenti lorsque l'étrange et l'insolite font irruption dans le texte et que le cadre réconfortant de la raison se lézarde irrémédiablement.

L'étude de Lise Morin est à la fois analogue et fort différente.
Comme Michel Lord, elle étudie la nouvelle, genre court ${ }^{5}$; de plus, son corpus est également tiré de la production de 1960 à 1985, restant ainsi fidèle aux bornes chronologiques posées par l'équipe de recherche dont ces volumes sont tributaires ${ }^{6}$. Le lecteur pourrait s'attendre à de nombreux recoupements mais, en réalité, ces livres sont différents et se complètent fort bien. Lise Morin étudie plus de nouvelles (seize au lieu de cinq); les textes sont plus présents, plus longuement analysés, et le passage des théories et des exposés conceptuels aux textes et à leur analyse est beaucoup plus rapide dans son cas que dans celui de Michel Lord. De plus, Lise Morin pratique souvent, et avec bonheur, une écriture tendant vers le ludique: apparemment désinvolte, surtout dans ses débuts de chapitre, mais cachant néanmoins sous ses libres associations et ses images enjouées unè grande cohérence de visée et une belle rigueur analytique.

Elle prend d'abord acte du changement important intervenu en littérature fantastique:

Car désormais la créature fantastique, qui aime à suivre la mode, a renoncé à ces toilettes tapageuses qui la faisaient repérer avec promptitude dans le blanc d'une page. Elle leur préfère bien souvent des tenues plus discrètes. De même, elle s'est lassée des cimetières sombres où l'on respire des parfums délétères et des châteaux menacés par le lierre et la foudre; aujourd'hui, elle fréquente volontiers les mêmes lieux que vous et moi. (14)

Cette constatation, dont le ton est bien indicatif de l'ensemble, entraîne deux conséquences. D'abord, puisque les apparences sont désormais 
trompeuses et que l'approche thématique ne suffit guère, il importe désormais de faire appel à des instruments d'analyse subtils: les dimensions discursive, narratologique et sémiotique. Ensuite, puisque le fantastique a changé et qu'on ne le reconnaît plus, il faut distinguer entre le fantastique canonique et le néo-fantastique:

Les textes qui ressortissent au fantastique canonique doivent leur dénomination à leur ressemblance avec certains récits fantastiques français du siècle dernier: ils font un sort à la figure du hérosvictime, pris dans une odieuse machination qui le laisse sans ressource et qui le conduit fatalement à sa perte. Ces écrits instaurent un climat de peur à grand renfort de visions cauchemardesques et se terminent le plus souvent sur une note dysphorique. Ce sont eux qui sont restés gravés dans l'imagination populaire. Quant aux récits néo-fantastiques, ils mettent en scène des personnages qui voient se fracturer leur quotidien de façon plus ou moins marquée. Ces personnages reconnaissent que les transformations qui s'opèrent sous leurs yeux dérogent aux règles normales - à défaut de quoi on passerait du fantastique au merveilleux - mais ne s'en formalisent pas outre mesure. En règle générale, l'issue ne leur est pas néfaste. Les auteurs de ces textes tablent volontiers sur l'humour, l'ironie. (22)

C'est le sens du sous-titre de l'étude et l'essentiel du propos du l'auteur: les écrits québécois contemporains oscillent entre les deux registres.

L'introduction, outre les précisions essentielles sur l'évolution du genre, l'importance de la production, le choix du corpus, la méthode d'analyse et le plan de l'ouvrage, présente aussi, à grands traits, une explication intéressante de la disparition puis du retour du fantastique, complétant ainsi fort bien les propos de Michel Lord. La résurgence du fantastique, depuis 1960, serait liée à l'émergence d'une nouvelle rationalité et à l'apparition d'une conscience schizoïde, reflet d'une vision problématique du réel, et rendue possible par l'effritement du pouvoir clérical monolithique. En somme, 1960 reproduit 1770: la foi est ébranlée, mais la nouvelle rationalité ne dissipe pas tous les doutes ni les croyances séculaires en quelque forme de surnaturalisme. L'étrange, l'étonnant, l'incompréhensible succèdent aux dévotions.

Le premier chapitre de Lise Morin, comme celui de Michel Lord, mais de façon plus complète, effectue un survol des théoriciens du genre, examine leurs définitions et leurs méthodes d'analyse, les rattache à de grands schémas explicatifs; il effectue aussi toute une série de distinctions: entre le fantastique et les genres voisins (merveilleux, sciencefiction); entre les deux registres du fantastique (canonique, néo-fantastique); entre le conte et la nouvelle. Les distinguos succèdent aux rapprochements contrastés qui cèdent la place aux définitions qui entraînent les classifications. De fait, sur la foi de ces deux études, un lecteur peu averti serait justifié de croire que le premier devoir d'un critique du fantastique est de se livrer, pieds et poings liés, à une sorte de fureur taxinomique qui occupera entre le tiers et la moitié de son propos. Cela plaira aux nominalistes et aux friands de scientificité littéraire. Quant aux autres, ils n'y verront que les indices d'un discours, justifié dans le contexte, qui cherche à affirmer le 
dynamisme d'un genre et la pertinence d'un champ d'investigation.

Les trois chapitres analytiques du livre de Lise Morin étudient les * mille petites impostures qui émaillent le discours fantastique "et qui constituent le - traquenard où le lecteur est invité à perdre non pas son âme comme dans les pactes diaboliques, ni même son esprit, mais seulement ses certitudes * (79). Sont donc étudiés les * procédés mis en cuvre dans le texte pour conférer une certaine plausibilité à la narration de faits improbables " (80). Avec toujours, à l'esprit, la distinction entre les deux registres, mais aussi la conscience de ce qu'ils partagent. Le chapitre II isole certaines unités élémentaires (marqueurs, modélisateurs, connotateurs de fantasticité, ces derniers étant de type cognitif ou affectif), s'interroge sur l'ironie et la présence d'un - personnage rationnel ", et affirme que "l'armature fantastique. (97) se trouve dans la narration, la focalisation et la représentativité logique; non pas que ces composantes soient propres aux œuvres fantastiques: elles y ont simplement une importance exceptionnelle. Le troisième chapitre prolonge cette analyse en réintégrant la temporalité dans le texte: de la grammaire du texte on passe à sa syntaxe, c'est-à-dire à l'examen des enchaînements narratifs. Le quatrième chapitre interroge lidéologie à l'œuvre dans les récits. Après avoir déterminé trois champs de normes qui peuvent être transgressés par le fantastique (le code logique, le code culturel, le code littéraire), Lise Morin effectue un certain nombre d'observations fondamentales. D'abord que le code n'est pas présent dans les textes: il n'est donné que par son envers, son infraction. *Aussi doit-on reconstruire la règle à partir de la perturbation." (194) Ensuite que les récits violent le code logique (ce qui était prévisible: c'est pour cela qu'ils sont étranges, mystérieux). Finalement qu'ils confortent l'idéologie dominante et ne remettent aucunement en question l'ordre social, ni les valeurs et les habitus: :[E]n dépit de l'extravagance de leur fabula, les cuvres fantastiques reposent assez souvent sur une idéologie conventionnelle. " (221) À la lumière de ces constatations, on est quelque peu étonné, à la fin de ce chapitre, lorsque l'auteure affirme que le fantastique :fait éclater ", "dénonce ", subvertit " non seulement l'illusion référentielle, mais *le mythe de la signification . (254). C'est accorder beaucoup d'importance, il me semble, à des œuvres qui ont moins de prétention et dont l'effet, fort limité dans le temps - à peine le temps de la lecture, et même moins, dans la plupart des cas, car ils sont rares les fantastiqueurs qui réussissent à bien accrocher le lecteur - ne dépasse guère le simple plaisir d'être étonné, surpris, inquiété, avant de passer aux choses sérieuses: au quotidien beaucoup trop réel, d'une part, mais aussi aux œuvres qui ont plus de poids...

\section{**}

Ces lectures auront-elles fait de moi ou feront-elles des autres sceptiques, indifférents au genre, des fervents du fantastique? Cela serait étonnant. D'ailleurs, telle n'est pas l'intention des auteurs. Ces deux études participent de l'intérêt actuel, massif, pour toutes les formes de 
paralittérature, allant du roman Harlequin aux récits de science-fiction. À un certain niveau, on pourrait s'interroger sur le déploiement d'une si grosse machinerie théorique pour étudier des œuvres restreintes, courtes et d'une importance relative. Par ailleurs, il est indéniable que la mise à l'essai de ces méthodes d'analyse et l'effort de systématisation et de conceptualisation qui en découle ne peuvent que contribuer à notre connaissance du fonctionnement de la mécanique textuelle. Nous voici donc, au terme de ces lectures, plus sensibles aux multiples procédés par lesquels les textes narratifs construisent le sens, ou le font basculer.

1. Dont le groupe de l'Université Laval, dirigé par Maurice Émond et Aurélien Boivin, à l'origine de nombreux travaux, thèses et mémoires, et en particulier des deux études dont il est ici question.

2. Michel Lord, La logique de l'impossible. Aspects du discours fantastique québécois, Québec, Nuit blanche, 1995, 361 p.
3. Lise Morin, La nouvelle fantastique québécoise de 1960 à 1985 . Entre le hasard et la fatalité, Québec, Nuit blanche, 1996, $301 \mathrm{p}$.

4. Curieusement, c'est dans une note (p. 15) que le lecteur apprend que les récits étudiés seront des nouvelles, exclusivement.

5. Elle annonce, toutefois, qu'elle abordera - ailleurs les différences entre le roman fantastique et la nouvelle fantastique. (p. 20, note 8).

6. Ce qui n'est pas sans poser un certain problème au lecteur. La période 19601985 est celle du recensement de Boivin, Émond et Lord (voir Morin, p. 20, note 9). Mais comme la période subséquente (post 1985) est celle de -l'institutionnalisation du fantastique et de la SF au Québec. (Lord, p. 18, note 12), il est étonnant que le corpus retenu, dans un cas comme dans l'autre, n'inclue aucune œuvre de cette dernière période. Est-ce que la période d'institutionnalisation est une période de pénurie? La clôture de 1985 se comprend dans le cas d'une équipe de recherche qui commence son travail en 1986, mais moins pour des auteurs qui signent des livres en 1995 et 1996 . Lise Morin, en particulier, dont l'étude cerne avec précision les caractéristiques du néofantastique, aurait eu tout intérêt, il me semble, à aborder les œuvres les plus récentes. 